\title{
Analysis of Digital Library System Based on Personalized Service
}

\author{
Min Fu \\ Jiangsu Vocational Institute of Commerce, Nanjing, China. \\ 1982fumin@163.com
}

\begin{abstract}
With the rapid development of information technology, the digital library came to the daily life, which greatly simplifies the difficulty of people's information retrieval and enables the knowledge to pass regardless of time and space constraints. Nowadays, the third-generation digital library, which is characterized by personalized services, has become the mainstream and meets the diverse needs of users. This paper firstly analyzes the concept and characteristics of digital library, then elaborates on the inevitability of the birth of personalized service and the necessity of its development and explains how to implement this personalized service from three aspects, summary the digital library based on personalized service and elaborated the future development trend of the digital library in the end.
\end{abstract}

Keywords: Digital Library; Personalized Service; Information Technology; User-oriented.

\section{基于个性化服务的数字图书馆系统分析}

\author{
傅敏 \\ 江苏经贸职业技术学院, 南京 中国.
}

摘 要: 随着信息化技术的快速发展, 催生了数字图书馆的诞生, 极大的简化了人们信息检索 的难度, 使知识能够穿越时间和空间的限制。现在, 以个性化服务为主要特点的第三代数字 图书馆已经成为当今时代的主流，满足了用户的多样化需求。本文首先分析了数字图书馆的 概念和特点, 随后阐述了个性化服务诞生的必然性以及其发展的必要性, 并从三个方面说明 了如何具体实施这种个性化服务, 最后对基于个性化服务的数字图书馆进行了总结, 并阐述 了数字图书馆未来的发展趋势。

关键词: 数字图书馆; 个性化服务; 信息技术; 用户为中心.

\section{1. 前言}

随着科学技术的高速发展, 以计算机技术和通信技术为基础的互联网将人们带入了现代信息 社会。与此同时, 在传统的图书馆分类管理系统逐渐不能适应人们日益增长的文化需求之后, 图书馆系统进入了第三代，即数字图书馆时代，它具有分布式、异构化和个性化的特点 [1]。 根据数字图书馆的这些特点和信息资源激增的大背景, 学者必须开展研究以用户为中心的个 性化数字图书馆服务技术。它强调以人为本，为用户提供个性化的信息，并节省他们的信息 搜索时间。研究图书馆的个性化服务, 就是研究如何使其能够有效地组织个性化信息, 对用 户提供主动、互惠、个性化的服务, 满足用户的多样性需求。

由于数字图书馆的重要性, 国内外许多不同学者都对这个领域进行了相关的研究。国外学者 Ekere 等人研究了用户对数字图书馆的设施、资源和服务的认知程度 [2]。通过调研, 他们发 现用户对数字图书馆的资源有很清晰的认知度, 如网络数据库、门户网站、在线摘要等。但 是同时他们也强调现在的数字图书馆在用户个性化服务方面做得不够, 并呼吁加强这方面的 研究。另一方面, 国内研究者赖远根等人着力研究数字图书馆的客户流失情况 [3]。通过他们 的研究发现, 数字图书馆服务存在很高的客户流失率, 当客户在图书馆的网站上注册后的三 个月内, 给定图书馆的客户流失率相当大。分析客户的流失原因发现, 数字图书馆系统未能 
很好的针对客户进行个性化服务, 且不同于传统的图书馆可能会有人与人之间的交流, 客户 现在面对的只是一个冰冷的机器而已。所以, 我们可以发现数字图书馆虽然不断发展, 技术 不断革新, 但是在某种程度上, 它只是一种针对单一客户和客户群体的静态服务理念, 未能 根据客户实际情况提供个性化服务。因此, 进行数字图书馆的个性化服务是势在必行的。

\section{2. 数字图书馆个性化服务及其模型分析}

2.1. 数字图书馆及其特征分析

数字图书馆及其相关技术正在逐步进入每个人的生活中, 变成人们日常工作学习不可或缺的 工具。但是要对数字图书馆进行有效的定义却不是那么的容易。数字图书馆的概念一开始是 由一些物理科学家所提出的, 而不是由图书馆管理员率先发明的。在万维网技术普及之后, 各种版本的图书管理软件也逐渐涌现出来。现在, 一个广受大众接受的对数字图书馆的定义 是 $[4]$ : 数字图书馆是一个信息系统, 它可以提供大量的、有组织的信息和知识库, 便于用户 进行搜索和使用。

在了解了数字图书馆的定义之后, 我们必须清楚的了解其特点, 这是由于数字图书馆的这些 特点决定了相关技术的实现和个性化服务的质量。随着图书馆的建设和发展, 作为服务于人 的数字图书馆, 首先必需具备以下几个特点: 信息资源数字化、信息获取网络化、基于 IT 技 术的信息资源分享。除此之外，还有一些其他特征。

(1) 元数据: 在早期的数字图书馆研究中, 所面临的一个问题是如何制作图书目录、分类和材 料来源说明, 也就是如何定义元数据, 以便让用户更容易的查找 [6]。传统的数字图书馆使用 列表、摘要、目录等来解决这个问题。因此, 数字图书馆的一个最大特征就是元数据的问题。 (2) 分布式: 数字图书馆管理信息数据的方式有很多种, 但是将来希望最大的可能就是基于分 布式管理的数字图书馆系统。

(3) 资源调度: 数字图书馆系统通过分析单位的位置, 将不同单位的分布式资源和公共资源分 发出去, 而传统图书馆资源的资源调到主要是取决于它们合同, 如 Z39.2 合同 [7]。如果它们 之间没有合同, 即使达成了协议, 它们也不能来分享资源。因此, 在使用数字图书馆的时候, 进行合理的资源调度是至关重要的。

\section{2. 数字图书馆个性化服务分析}

个性化服务概念的出现最早可以追溯到经济领域。在经历了计划经济的时代之后, 自我中心 的理论被当今网所淹没, 取而代之的是以客户为中心的理论。计划经济时代的推广服务的概 念也被新的个性化服务理念所替代。与推广服务相比, 个性化服务是一种为不同客户提供不 同服务策略、不同服务内容和不同服务功能的新型模式 [8]。它有三个层面的隐形要求:第一, 每个客户都要有自己的知识需求; 第二, 作为服务的提供者, 需要有一个明确的目标, 并可 以对结果进行检验; 第三，服务的提供者和接受者应该能相互合作，使双方都感到满意。

个性化服务的理念以用户为中心, 倡导以人为本的思想, 因此对数字图书馆的建设具有重要 的意义。其重要性主要体现在以下三个方面:

(1) 数字图书馆的可持续发展需要个性化的服务, 甚至趋向于传统图书馆和图书馆的复合发 展模式。

(2) 数字图书馆将面向公众的模式转变为面向个人。一方面可以为用户节省时间, 另一方面 有助于培养个性化, 引导需求, 最终促进社会向多元化去发展。

(3) 数字图书馆以用户为中心, 优化了图书馆信息的分布, 将知识经济的理念应用于图书馆 的建设, 能够发挥其功能, 提高质量和效率。

2.3. 数字图书馆个性化服务的实现

有关数字图书馆的的创造性项目已经由美国科学自然基金协会发起, 这个项目最重要的一点 就是对用户的个性化服务的研究, 以帮助用户能够高效的创建、查询和利用知识信息, 并促 进相关数字图书馆技术的发展。个性化服务的实施在这一种过程起到至关重要的作用, 因此 必须有效的对个性化服务进行落地实施。 
为了推进数字图书馆个性化服务的实施，必须加快对数字图书馆资源的开发。随着数字图书 馆的相关技术的逐渐成熟, 已经形成了庞大的系统结构, 为用户带来了大量丰富的信息。由 于用户对信息的需求是有限的, 大多数情况下他们的兴趣非常稳定。所以, 个性化服务必须 围绕用户能够高效的利用这一知识来源为核心进行展开, 即图书馆信息资源。对于用户来说, 他们可以根据自己意愿创建需要的文件, 并上传他们的信息资源。这样系统便能结合他们感 兴趣的信息库进行个性化分析，使得他们在以后的检索过程中很快便能得到自己想要的信息。 而且, 这个过程还丰富了系统的资源, 可以采用大数据和人工智能技术对用户上传的自己感 兴趣资源进行分类 [9], 并打好标记, 为以后的相关功能展开做好铺垫。为了这一过程能够顺 利实施，必须要利用众包技术来丰富系统的资源库 [10]。

数字图书馆的本质上是为了满足用户信息需求而产生的, 使用户在搜索信息的时候减少了许 多的麻烦。然而, 随着信息的急剧增加, 进行信息检索的难度会增大。这是因为过多的额外 信息需要他们进行判断和去除, 这个过程需要一些时间消耗。因此, 数字必须要采用合理的 检索方式来满足用户的这个需求。根据检索的原始数据, 并结合用户感兴趣的知识, 可以检 索出他们需要的信息, 并采用适当的方式进行展示出来 [11]。因此, 为了高效的实施数字图 书馆的个性化服务必须采用合理的检索策略。

除了以上两种措施之外, 为了更好提高数字图书馆的服务质量, 还需要开发出精确的推荐系 统以用来向客户推荐合适的信息。对用户的推荐服务是即时个性化服务的体现, 它是以用户 感兴趣的知识库为中心, 以最新的数据源为基础, 以信息技术为动力主动向客户提供个性化 服务的措施。因此, 为了能很好的进行信息推荐, 满足用户的潜在需求, 必须有充足的数据 源, 这就需要用户的前期参与。在收集好相关的数据后, 便要采用合适的推荐技术进行分析 数据 [12], 并给出合理的结果, 这一过程是至关重要的。如图 1 所示, 它展示了基于推荐系 统的数字图书馆个性化服务的一般信息处理流程。

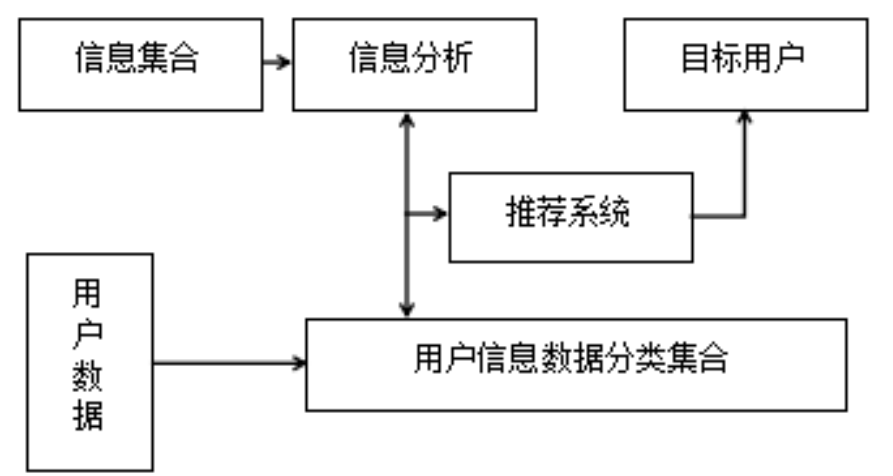

图 1.基于推荐系统的数字图书馆个性化服务信息处理流程

\section{3. 结论}

随着全球科技竞争的加剧, 对于数字图书馆的研究已经成为这次科技竞赛的重要赛点, 它也 是评估一个国家信息基础设施的重要标志之一。由于数字图书馆在很大程度上决定了整个科 学发展的关键节点, 对国民的知识储备起到极大的作用, 所以它对于整个社会的可持续发展 有很大的影响力, 决定了整个社会的未来走向。所以我们必须加大对数字图书馆这一方面的 研究, 以来促进整个国家的软实力的发展。其中, 在数字图书馆的研究中, 能够进行未来方 向准确的预判并赶在其他国家之前快速的实施至是关重要的。

国内外学者的许多研究都表明, 面向用户和服务的数字图书馆模式将成为未来发展的主要趋 势。这种模式极大地关注人们对数字图书馆的感受, 并围绕信息系统和用户的关系, 对用户 提供精确的服务。它强调针对用户的个性化服务, 以此来减少用户的时间和精力的不必要浪 费。本文首先分析了数字图书馆的概念及其三个主要的特征, 随后对数字图书馆的个性化服 务进行探讨, 并从三个方面论述了其重要性, 最后从理论层面上分析如何具体落地实施数字 
图书馆的个性化服务，主要包括三个方面：加大图书馆资源的开发、采用合理的检索技术以 及利用推荐系统进行精准信息推送。值得一提的是, 由于人工智能和数据挖掘的强大力量, 以及它们与图书馆技术有着千丝万缕的联系, 将这些技术引入图书馆的研究中将会可能得到 意想不到的的成果, 这也是值得未来研究者所需要关注的。在明确了未来发展的方向以及具 体实现方式之后, 必须要加大研究力度, 使新的数字图书馆系统能够真正做到智能化和精准 化, 为用户提供更优质的服务, 为科技的发展做好基础工作, 将国家和科技进步带来的成果 真正惠及到每一个公民。

\section{References}

[1]. Saracevic T. Digital library evaluation: Toward an evolution of concepts[J]. 2000.

[2]. Nwoha, Chidinma M. "USERS'PERCEPTION OF THE FACILITIES, RESOURCES AND SERVICES OF THE MTN DIGITAL LIBRARY AT THE UNIVERSITY OF NIGERIA, NSUKKA." Library philosophy and practice (2016): 1.

[3]. Lai, Yuangen, and Jianxun Zeng. "Analysis of customer churn behavior in digital libraries." Program 48.4 (2014): 370-382.

[4]. Zhangzheng, "The Overall Statement About Digital Library in New Century", The Theory and Practice About Library.2004(1).

[5]. Hong, W., Thong, J.Y., Wong, W.M. and Tam, K.Y., 2002. Determinants of user acceptance of digital libraries: an empirical examination of individual differences and system characteristics. Journal of Management Information Systems, 18(3), pp.97-124.

[6]. Beall, J., 2006. Metadata and data quality problems in the digital library. Journal of Digital Information, 6(3).

[7]. Lynch, C.A., 1991. The Z39. 50 information retrieval protocol: An overview and status report. ACM SIGCOMM Computer Communication Review, 21(1), pp.58-70.

[8]. Renda, M.E. and Straccia, U., 2005. A personalized collaborative digital library environment: a model and an application. Information processing \& management, 41(1), pp.5-21.

[9]. Chang, C.C. and Chen, R.S., 2006. Using data mining technology to solve classification problems: A case study of campus digital library. The Electronic Library, 24(3), pp.307-321.

[10]. Holley, R., 2010. Crowdsourcing: How and why should libraries do it. D-Lib Magazine, $16(3 / 4 \mathrm{Ma})$.

[11]. Kowalski, G.J., 2007. Information retrieval systems: theory and implementation (Vol. 1). Springer.

[12]. Avancini, H., Candela, L. and Straccia, U., 2007. Recommenders in a personalized, collaborative digital library environment. Journal of Intelligent Information Systems, 28 (3), pp .253-283. 